\title{
EXTREMELY AMENABLE SEMIGROUPS ${ }^{1}$
}

\author{
BY E. E. GRANIRER
}

Communicated by V. Klee, July 8, 1966

Let $S$ be a discrete semigroup and $m(S)$ the Banach space of bounded real functions on $S$ with the usual norm $\|f\|=\sup \{|f(s)|$; $s \in S\}$. A linear functional $\phi$ on $m(S)$ is a mean if $\phi(f) \geqq 0$ for $f \in m(S)$ with $f \geqq 0$ and $\phi(1)=1$, where 1 is the constant one function on $S$.

$\mathrm{S}$ is extremely left amenable (ELA) if there exists a multiplicative left invariant mean $\phi$ on $m(S)$, i.e. a mean $\phi$ which satisfies $\phi(f g)=\phi(f) \phi(g)$ for any $f, g \in m(S)$ and $\phi\left(f_{a}\right)=\phi(f)$ for each $f \in m(S)$ and $a \in S$ (where $f_{a}(s)=f(a s)$ and $f^{a}(s)=f(s a)$ for any $a, s \in S$ and $\left.f \in m(S)\right)$.

The first to consider ELA semigroups (under different terminology) was T. Mitchell in [13]. His main and interesting result in [13] is that a semigroup $S$ is ELA if and only if it has the common fixed point property on compacta (i.e. for each compact hausdorff space $X$ and for each homomorphic representation $S^{\prime}$ of $S$ as a semigroup (under functional composition) of continuous maps of $X$ into itself, there is some $x_{0}$ in $X$ such that $s^{\prime}\left(x_{0}\right)=x_{0}$ for all $s^{\prime}$ in $S^{\prime}$ ). This result is an analog of Day's generalisation of the Markoff Kakutani fixed point theorem [4].

Let $m(S)^{*}$ be the conjugate Banach space of $m(S)$. If $\phi \in m(S)^{*}$, let $\left(L_{a} \phi\right)(f)=\phi\left(f_{a}\right)$ for any $f \in m(S)$ and $a \in S$. Also let $1_{a} \in m(S)^{*}$ be defined by $1_{a}(f)=f(a)$ for $f \in m(S)$ and $a \in S$. Elements of $\left\{1_{a}\right.$; $a \in S\}$ are called point measures. $\beta(S) \subset m(S)^{*}$ denotes the set of multiplicative means. $\beta(S)$ becomes a semigroup, which contains $S$, under the convolution multiplication: $\phi \odot \psi(f)=\phi(g)$ where $g(s)$ $=\psi\left(f_{s}\right)$ for $s \in S$.

Define $r_{a}, l_{a}: m(S) \rightarrow m(S)$ by $r_{a} f=f^{a}, l_{a} f=f_{a}$ for $a \in S$. If $f \in m(S)$, denote by $K(f)$ the set of reals $c$ for which there is some net in $\left\{r_{a} f\right.$; $a \in S\}$ which converges poin twise to the constant function $c \cdot 1(1 \in m(S)$ is the constant one function on $S$ ). $S$ is extremely right stationary if $K(f) \neq \varnothing$ for each $f \in m(S)$. (Compare with Mitchell [12, p. 246]).

Let $A \subset m(S)$ be a uniformly closed left invariant (i.e., $f_{s} \in A$ for any $s \in S$, if $f \in A$ ) subalgebra with $1 \in A$. Denote by $H_{A}$ the ideal of all $h \in A$ such that $h=\sum_{1}^{n} f_{j}\left(g_{j}-l_{a_{j}} g_{j}\right)$ for some $f_{j}, g_{j} \in A$ and some $a_{j} \in S, 1 \leqq j \leqq n$ where $n=1,2, \cdots . K_{A}$ will denote the linear sub-

1 The results of this note will appear in two papers entitled Extremely amenable semigroups and Extremely amenable semigroups. II in Math. Scand. The first paper was partially supported by research grant Nonr 401(50) at Cornell University. 
space of all $h \in A$ with $h=\sum_{1}^{n}\left(g_{j}-l_{a_{j}} g_{j}\right)$ for some $g_{j} \in A, a_{j} \in S$, $1 \leqq j \leqq n$ and $n=1,2, \cdots$. In case $A=m(S)$ we denote $H_{A}=H$ and $K_{A}=K$. A linear functional $\phi$ on $A$ is a mean if $\phi(1)=1$ and $\phi(f) \geqq 0$ for $f \geqq 0, f \in A$. The algebra $A$ is said to be ELA if there exists a multiplicative mean on $A$ which is left invariant. Hence $S$ is ELA iff the algebra $m(S)$ is ELA.

We have the following characterisation of ELA semigroups:

THEOREM A. Let $S$ be a semigroup. The following conditions on $S$ are equivalent:

1. $S$ is ELA.

2. There is a net of point measures $\left\{1_{s_{\alpha}}\right\}$ such that $\lim \left\|L_{a} 1_{s_{\alpha}}-1_{s_{\alpha}}\right\|$ $=0$ for each $a \in S$.

3. Each two elements of $S$ have a common right zero, i.e. for $a, b \in S$ there is some $c \in S$ with $a c=b c=c$. (Hence no nontrivial right cancellation ELA semigroups exist.)

4. $\beta(S)$ has a right zero (though $S$ does not need to have one).

5. $S$ is extremely right stationary and in this case $K(f)=\{\phi(f)$; $\phi$ ranges over all multiplicative left invariant means on $m(S)\}$.

6. For each $h \in H$ there is some $s \in S$ such that $h(s)=0$ (there still may be some $h$ in the uniform closure of $H$ with $h(s) \neq 0$ for any $s \in S$, even though $S$ is ELA).

7. $S$ is left amenable and each left invariant mean $\mu$ on $m(S)$ satisfies $\mu\left(f g_{s}\right)=\mu(f g)$ for any $f, g \in m(S)$ and any $s \in S$.

8. $S$ is left amenable and each extreme point of the set of left invariant means on $m(S)$ is multiplicative.

9. $S$ is left amenable and $K$ is uniformly dense in $H$.

10. $S$ has the common fixed point property on compacta.

As we said, the interesting implication $(1) \Leftrightarrow(10)$ is due to Mitchell [13]. Furthermore, due to him is also $(3) \Rightarrow(1)$. Moreover, Mitchell proves $(1) \Rightarrow(3)$ in $[13]$ under additional assumptions on the semigroup $S$. In the present generality $(1) \Rightarrow(3)$ disproves a conjecture of Mitchell made in [13]. (1) $\Leftrightarrow(2)$ is a Day-Folner type characterisation of ELA semigroups (see Day [3, pp. 524-525] and Folner [7]). $(1) \Leftrightarrow(5)$ is in analogy to Mitchell's result in [12] for the left amenable case. His proof though, does not carry over to the extreme right stationary case. Conditions (6), (7), (8), (9) do not have analogues to left amenable semigroups.

For subalgebras $A \subset m(S)$ we have the following:

Theorem B. Let $A$ be a uniformly closed subalgebra of $m(S)$ with $1 \in A$. The following conditions on $A$ are equivalent:

1. $A$ is ELA, 
2. $\inf \left\{\|1-h\| ; h \in H_{A}\right\}=1$,

3. $\sup \{h(s) ; s \in S\} \geqq 0$ for each $h \in H_{A}$,

4. $H$ is not uniformly dense in $A$,

5. There is a mean $\phi$ on $A$ such that $\phi\left(f g_{x}\right)=\phi(f) \phi(g)$ for each $f, g \in A$ and $x \in S$.

Conditions (1)-(4) are Day-Dixmier type characterisations of ELA subalgebras $A \subset m(S)$ (compare with M. Day [2, pp. 281-282 and p. 286] and Dixmier [5, pp. 214-215] or with Theorems 17.4 and 17.15 in Hewitt-Ross [10]). For Condition 5 compare with the recent work of S. P. Lloyd [11].

We have the following application to topological groups: Let $G$ be an abelian topological group (not necessarily locally compact) and $C=C(G)[U=U C(G)]$ be the space of real bounded [uniformly] continuous functions on $G$. Assume in addition that $G$ has a nontrivial homomorphic image $G^{\prime}$ which is a subgroup of a locally compact abelian group. We show for this case that $U C(G)$ and afortiori $C(G)$ are not ELA. Applying this result together with Theorem B and the above quoted theorems of Day and Dixmier one gets the:

TheOREM. Let $G$ be an abelian topological group which has a nontrivial homomorphic image $G^{\prime}$ which is a subgroup of a locally compact abelian group. Then

(a) $U C(G)$ and $C(G)$ are not ELA even though both admit an invariant mean.

(b) $H_{U}\left[H_{C}\right]$ is uniformly dense in $U C(G)[C(G)]$ while $K_{U}\left[K_{C}\right]$ is not uniformly dense in $U C(G)[C(G)]$.

(c) $\sup \{h(x) ; x \in G\} \geqq 0$ for each $h \in K_{C}$ while $\sup \{h(x) ; x \in G\}<0$ for some $h \in H_{U}$.

(d) $\inf \left\{\|1-h\| ; h \in H_{U}\right\}=0$ while $\inf \left\{\|1-h\| ; h \in K_{C}\right\}=1$.

Some refinements of this theorem are also obtained.

We also study the class of ELA semigroups and get the following results which have known analogues to amenable semigroups:

(a) If $S$ is ELA so is any homomorphic image (due to Mitchell and immediate from Theorem A (1) $\Leftrightarrow(3))$.

(b) If $S$ is a semigroup with $S=U_{t \in T} S_{t}$ where $S_{t}$ are ELA semigroups and for any $t_{1}, t_{2} \in T, S_{t_{1}} \cup S_{t_{2}} \subset S_{t_{3}}$ for some $t_{3} \in T$ then $S$ is ELA (compare with Day [3, p. 516]).

(c) If $S$ is ELA then any countable subsemigroup is included in a countable ELA semigroup (compare with [8]).

(d) If $S$ is a semigroup and $I C S$ is a left ideal, then $S$ is ELA if and only if $I$ is ELA (compare with A. H. Frey [6] and Mitchell [12]). 
(e) If $S$ is ELA and $S_{0}$ a subsemigroup with $\phi\left(S_{0}\right)>0$ for some left invariant mean $\phi$ then $S_{0}$ is ELA (compare with Day [3, p. 518]). The analogy with amenable semigroups is though destroyed by

(f) If $S_{t}$ are ELA semigroups so is their full direct product. The analogue of $(f)$ to left amenable semigroups is not true (Day [3, p. $\left.\left.517 \mathrm{~F}^{\prime}\right]\right)$.

One can ask the following question in connection with (c):

If $S$ is ELA, is each finitely generated subsemigroup of $S$ included in a finitely generated ELA subsemigroup? This question is answered in the negative by an example of a semigroup $S$ with the following properties:

$S$ is a countable left cancellation ELA semigroup such that any subsemigroup which can be included in a finitely generated subsemigroup is not ELA while all those subsemigroups which cannot be included in finitely generated subsemigroups are ELA. Nevertheless, each subsemigroup of $S$ is left amenable and even admits an infinite dimensional set of invariant means (none of which is multiplicative if the subsemigroup is finitely generated). Furthermore, $S$ is not right amenable and does not contain elements of finite order.

In view of this example it is interesting to note that such a behaviour is impossible if $S$ contains "enough" periodic elements ( $c \in S$ is periodic if $c^{n}$ is an idempotent for some $n \geqq 1$ ). One has the

Proposition. Let $S$ be an ELA semigroup each of whose right ideals contains a periodic element. If $\left\{s_{1}, \cdots, s_{n}\right\} \subset S$ generate the subsemigroup $S_{0}$, then there is some $t \in S$ such that the subsemigroup generated by $\left\{s_{1}, \cdots, s_{n}, t\right\}$ is ELA.

We give in what follows a general construction for a big class of ELA semigroups. It comes out from this construction that any left cancellation semigroup can be embedded in a left cancellation ELA semigroup. This wealth of ELA semigroups seems to us surprising, in view of the fact that no right cancellation ELA semigroups exist (except the trivial $S=\{e\}$ with $e^{2}=e$ ). The above construction is specialised and studied in some detail. It gives rise for example to a left cancellation ELA semigroup $S$ such that if $\left\{s_{1}, \cdots, s_{n}\right\} \subset S$ generate the semigroup $S_{0}$ then there are $\{s, t\} \subset S$ such that the semigroup generated by $\left\{s_{1}, \cdots, s_{n}, s, t\right\}$ is not even left amenable while that generated by $\left\{s_{1}, \cdots, s_{n}, s\right\}$ is left amenable but not ELA.

We make in the end the following conjecture: Let $S$ be a left cancellation ELA semigroup which is also right amenable. Then $S$ is the trivial group containing identity only. If $S$ is extremely right amenable instead of being only right amenable, then the conjecture is 
clearly true from (3) of Theorem A applied to extremely right amenable semigroups. Furthermore this conjecture holds true if every element of $S$ has finite order (see [9, Lemma 4]) or even if $S$ has "enough" periodic elements. A stronger conjecture is due to $J$. Sorenson.

\section{REFERENCES}

1. M. M. Day, Ergodic theorems for abelian semigroups, Trans. Amer. Math. Soc. 51 (1942), 399-412.

2. - Means for the bounded functions and ergodicity of the bounded representations, Trans. Amer. Math. Soc. 69 (1950), 276-291.

3. —- Amenable semigroups, Illinois J. Math. 1 (1957), 509-544.

4. - - Fixed point theorems for compact convex sets, Illinois J. Math. 5 (1961), 585-590.

5. J. Dixmier, Les moyennes invariantes dans les semigroupes et leur applications, Acta. Sci. Math. (Szeged) 12 (1950), 213-227.

6. A. H. Frey Jr., Studies in amenable semigroups, Ph.D. Thesis, University of Washington, Seattle, Washington, 1960.

7. E. Folner, On groups with full Banach mean values, Math. Scand. 3 (1955), 243254.

8. E. Granirer, On amenable semigroups with a finite dimensional set of invariant means. II, Illinois J. Math. 7 (1963), 49-58.

9. - On the range of an invariant mean, Trans. Amer. Math. Soc. 125 (1966).

10. E. Hewitt and K. Ross, Abstract harmonic analysis, Springer, Berlin, 1963.

11. S. P. Lloyd, $A$ mixing condition for extreme left invariant means, (to appear).

12. T. Mitchell, Constant functions and left invariant means on semigroups, Trans. Amer. Math. Soc. 119 (1965), 244-261.

13. - - Fixed points and left invariant means. Trans. Amer. Math. Soc. 122 (1966), 195-202.

The University of British Columbia, Vancouver 